\title{
Comparison of Metabolic Dysfunction-Associated Fatty Liver Disease with Non-Alcoholic Fatty Liver Disease in Identifying Cardiovascular Risk in Chinese Individuals with Rheumatoid Arthritis
}

Lie Dai ( $\nabla$ dailie@mail.sysu.edu.cn )

Sun Yat-sen Memorial Hospital

Yao-Wei Zou

Sun Yat-sen Memorial Hospital

Qian-Hua Li

Sun Yat-sen Memorial Hospital

Jing-Wei Gao

Sun Yat-sen Memorial Hospital

Jie Pan

Sun Yat-sen Memorial Hospital

Jian-Da Ma

Sun Yat-sen Memorial Hospital

Le-Feng Chen

Sun Yat-sen Memorial Hospital

Jian-Zi Lin

Sun Yat-sen Memorial Hospital

Ying-Qian Mo

Sun Yat-sen Memorial Hospital

Xue-Pei Zhang

Sun Yat-sen Memorial Hospital

Pin-Ming Liu

Sun Yat-sen Memorial Hospital

\section{Research Article}

Keywords: Cardiovascular disease, Rheumatoid arthritis, Metabolic dysfunction-associated fatty liver disease, Non-alcoholic fatty liver disease

Posted Date: February 16th, 2022

DOI: https://doi.org/10.21203/rs.3.rs-1352019/v1

License: (c) (1) This work is licensed under a Creative Commons Attribution 4.0 International License. Read Full License 


\section{Abstract}

Background: The nomenclature from non-alcoholic fatty liver disease (NAFLD) to metabolic dysfunction-associated fatty liver disease (MAFLD) is considered to identify more cardiovascular disease (CVD) risk in general population, and patients with rheumatoid arthritis (RA) carry an excess risk for CVD. We aimed to compare MAFLD with NAFLD in identifying CVD risk in RA patients.

Methods: Clinical data from a Chinese RA cohort were retrospectively analyzed. Hepatic steatosis was defined by abdominal ultrasound examination. CVD risk in RA patients was estimated by the Prediction for Atherosclerotic Cardiovascular Disease Risk in China.

Results: Among 513 included RA patients, $78.4 \%$ were female and the mean \pm SD age was $51.8 \pm 12.6$ years. The prevalence of MAFLD and NAFLD was $21.4 \%$ and $20.5 \%$, respectively. $10.9 \%$ RA patients concomitated with CVD events and $32.4 \%$ had a high 10-year CVD risk. Multivariate logistic regression analysis showed that both MAFLD and NAFLD were associated with an increase in CVD events (MAFLD: AOR = 2.303; NAFLD: AOR = 2.478) and high 10-year CVD risk (MAFLD: AOR = 3.184; NAFLD: $A O R=2.870$, all $p<0.05$ ). The net reclassification index and integrated discrimination improvement indicated no additional CVD events and high 10-years CVD risk were identified when replacing NAFLD with MAFLD in RA patients.

Conclusions: Both MAFLD and NAFLD are associated with an increased CVD risk which implies the importance of early detection and management of MAFLD or NAFLD in RA patients. However, new nomenclature of MAFLD identify no additional CVD risk in RA patients.

\section{Background}

Rheumatoid arthritis (RA) is a progressive autoimmune disease characterized by persistent joint inflammation that causes joint damage, deformities and dysfunction. However, as a systemic inflammatory disease, extra-articular organ involvement is common in RA, such as cardiovascular, pulmonary, neurological, gastrointestinal, renal, and hematologic disease [1]. Since inflammation, a characteristic for RA, acts a central role in the development of cardiovascular disease (CVD), it is not surprising that RA patients have a higher risk of cardiovascular morbidity and mortality (e.g. myocardial infarction, stroke, heart failure, etc) [2, 3]. In general, about $50 \%$ of deaths among RA patients are attributable to CVD-related causes [3]. CVD mortality is increased by approximately $50 \%$ in patients with RA compared with the general population [4]. In addition to traditional CVD risk factors, RA disease characteristics may play roles in the development of CVD among RA patients. Thus, it is of great importance to identify the strong predictors of CVD and take an early intervention in RA.

Non-alcoholic fatty liver disease (NAFLD) has emerged as the most common-chronic liver disease worldwide [6]. NAFLD may progress to fibrosis, cirrhosis and hepatoma, resulting in a raised hazard for metabolic and CVD deaths [6, 7]. Metabolic dysfunction-associated fatty liver disease (MAFLD), a novel nomenclature changing from NAFLD in 2020, has its own inclusion criteria by adding overweight/obesity, type 2 diabetes mellitus (T2DM) and evidence of metabolic dysregulation based on the definitions of NAFLD [8]. The alteration from NAFLD to MAFLD was proposed to identity metabolic fatty liver regardless of alcohol intake or other concomitant liver diseases [8]. It has been suggested that the NAFLD-to-MAFLD alteration can recognize higher CVD risks in the general population [9].

However, the prevalence of MAFLD and its relationship with CVD risks in patients with RA have not been reported. Thus, we investigated the prevalence of MAFLD in patients with RA and conducted a cross-sectional analysis of whether the MAFLD characteristics identified additional CVD risks than NAFLD.

\section{Materials And Methods}

\section{Study design and participants}


This retrospective study was carried out based on our RA cohort [10-13] at Department of Rheumatology, Sun Yat-sen Memorial Hospital, China. Subjects > 16 years old with a confirmed diagnosis of RA (2010 criteria) [14] were recruited when they finished abdominal ultrasound examination from June 2015 to September 2021. Subjects with other autoimmune diseases, serious infection, malignancy and pregnancy were excluded. Ethical approval mandatory for this study was obtained from Ethics Committee at Sun Yat-sen Memorial Hospital (SYSEC-KY-KS-2020-208) along with an informed consent from each patient.

\section{Data collection}

Available demographic and clinical data were collected at enrollment as we previously reported [10-13]. RA disease activity was assessed using the clinical disease activity index (CDAI). Physical function was assessed with the Stanford health assessment questionnaire disability index (HAQ-DI). Conventional radiographs of bilateral hands and wrists (anteroposterior view) were assessed with the Sharp/van der Heijde modified score.

\section{Laboratory measurement}

Overnight fasting venous blood samples were collected. Liver biochemistry parameters and metabolic parameters (including alanine aminotransferase [ALT], aspartate aminotransferase [AST], $y$-glutamyl transferase [GGT], alkaline phosphatase [ALP], total bilirubin [TBIL], albumin [ALB], fasting plasma glucose [FPG], glycated hemoglobin [HbA1c], fasting insulin [FINS], total cholesterol [TC], triglyceride [TG], high-density lipoprotein cholesterol [HDL-C], low-density lipoprotein cholesterol [LDL-C]) were measured. Abnormal liver function tests (LFTs) were defined as at least 1 value higher than the upper limit of normal (ULN) for ALT, AST, GGT, ALP and/or TBIL [15]. The homeostasis model assessment of insulin resistance (HOMA-IR) was calculated to evaluate insulin resistance (IR) [16]. The fatty liver index (FLI) and non-invasive liver fibrosis models (including fibrosis-4 index [FIB-4], NAFLD fibrosis score [NFS] and Forns index) were evaluated by original formulas [6, 17]. Advanced fibrosis was defined by FIB-4 $\geq 1.3$ (age $<65$ years) or $\geq 2.0$ (age $\geq 65$ years), NFS $\geq-1.455$ (age $<65$ years) or $\geq 0.12$ (age $\geq 65$ years) and Forns index $\geq$ highest quartiles $[6,8,17]$.

\section{Assessment of NAFLD and MAFLD}

Hepatic steatosis was defined by abdominal ultrasound examination. NAFLD was diagnosed if a subject had steatosis without over alcohol intake ( $\geq 30 \mathrm{~g} / \mathrm{d}$ in men and $\geq 20 \mathrm{~g} / \mathrm{d}$ in women) or concomitant liver disease (e.g. viral hepatitis, autoimmune liver diseases, etc.) [6]. MAFLD was diagnosed if a subject had hepatic steatosis with one or more of the three criteria: overweight/obese (body mass index $[\mathrm{BMI}] \geq 23 \mathrm{~kg} / \mathrm{m}^{2}$; by the Asia-Pacific criteria), T2DM and metabolic abnormalities described by at least any two indicators: (1) waist circumference (WC) $\geq 90 \mathrm{~cm}$ in men and $\geq 80 \mathrm{~cm}$ women; (2) blood pressure (BP) $\geq 130 / 85 \mathrm{mmHg}$ or taking anti-hypertension drugs; (3) TG $\geq 150 \mathrm{mg} / \mathrm{dL}$ or taking lipid-lowering agents; (4) HLD-C < 40 mg/dL in men and < 50 mg/dL in women or taking lipid-lowering agents; (5) Prediabetes (e.g., FPG

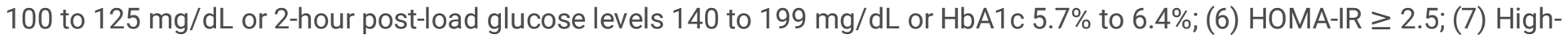
sensitivity C-reactive protein (hs-CRP) level $>2 \mathrm{mg} / \mathrm{L}$ [8]. In this study, we had no data of hs-CRP. CRP (scatter turbidimetry method, Siemens Healthcare Diagnostics, range: 0-5 mg/L) was used to instead of hs-CRP, because CRP was recommended as a disease activity indicator by ACR and EULAR guideline for RA management [14] and the elevated CRP was defined as $\mathrm{CRP}>5 \mathrm{mg} / \mathrm{L}$.

\section{CVD events and 10-year CVD risk estimation}

CVD events were defined as a verified medical history of coronary, cerebral and peripheral arterial disease, included angina pectoris, heart failure, myocardial infarction, ischemic or hemorrhagic stroke and peripheral arterial disease [3]. CVD events 
were collected by a questionnaire survey combined with verification through medical records.

The 10-year CVD risk of RA patients was assessed by the Prediction for Atherosclerotic Cardiovascular Disease (ASCVD) Risk in China (China-PAR) [18], Systemic Coronary Risk Evaluation (SCORE) for the low and high-risk regions in Europe [19] and Framingham Risk Score (FRS) [20]. The 10-year CVD risk estimation for RA patients was performed using a 1.5 multiplication factor to the risk estimate by the CVD risk calculator used in the general population according to the EULAR recommendation [3], and were classified into low, moderate and high risk according to the Chinese guidelines on the primary prevention of cardiovascular diseases [21].

\section{Statistical analysis}

Patient characteristics were summarized as mean \pm standard deviations, medians (interquartile range) or frequencies (\%) where applicable. We used $t$-test, Mann-Whitney $U$ test, $\chi^{2}$ test or Fisher's exact tests when appropriate, to determined differences between groups. The 10-year CVD risk were transformed into dichotomous variables (low-moderate-risk and highrisk). Logistic regression modeling was conducted to examine the estimate odds ratio (OR) for the MAFLD and NAFLD in relation to risk of CVD events and high 10-year CVD risk. Net reclassification index (NRI) and integrated discrimination improvement (IDI) were applied to compare the diagnostic accuracy of MAFLD with NAFLD in identifying CVD events and high 10-years CVD risk. SPSS and R statistical software were used for all analyses. A two-sided $p$ value of $<0.05$ was considered statistically significant.

\section{Results}

\section{Demographic and clinical characteristics of all RA patients}

Among 755 enrolled RA patients, 242 who had missing laboratory data or abdominal ultrasound examination were excluded. Thus, a total of 513 RA patients were qualified for statistical analysis. As shown in Table 1, 78.4\% included RA patients were female, with mean age of $51.8 \pm 12.6$ years old. The median disease duration was 60 months (IQR 21 to 120 months). According to CDAl, 85.4\% RA patients were active (CDAl > 2.8), while $14.6 \%$ were remission (CDAl $\leq 2.8$ ). Moreover, $27.5 \%$ RA patients haven't received any previous glucocorticoid or DMARDs therapy for six months before enrollment (treatment naïve).

\section{Prevalence of MAFLD and NAFLD in RA patients}

The prevalence of MAFLD and NAFLD was 21.4\% (110/513) and 20.5\% (105/513) respectively. Specifically, 19.9\% (102/513) RA patients fulfilled both criteria of NAFLD and MAFLD. Eight (1.6\%) RA patients with chronic hepatitis B infection who were not classified as NAFLD were newly identified as MAFLD, while $3(0.6 \%)$ RA patients with NAFLD were not complicated with metabolic abnormalities. There was no significant difference of MAFLD or NAFLD prevalence between male and female RA patients (MAFLD: $22.5 \%$ vs. 21.1\%, $p=0.754$; NAFLD: $20.7 \%$ vs. 20.4\%, $p=0.941$, Figure 1A). After adjustment for the age and sex composition ratio according to the data in the 2020 China Statistical Yearbook [22], the standardized prevalence of MAFLD and NAFLD in our RA cohort was $20.5 \%$ and $19.4 \%$, respectively. The males had a higher standardized prevalence of MAFLD $(25.4 \%$ vs. $15.6 \%, p=0.020)$, and a higher tendency of standardized prevalence of NAFLD $(23.4 \%$ vs. $15.4 \%, p=$ 0.050 , Figure $1 \mathrm{C})$ than the females.

The prevalence of MAFLD increased with age, and RA patients at 50-59 years had the highest prevalence (28.5\%), followed by $60-69$ years $(23.3 \%)$ and $40-49$ years $(21.5 \%$, Figure $1 B)$. RA patients at $50-59$ years had the highest standardized prevalence of MAFLD (27.2\%), followed by $40-49$ years (25.7\%) and 60-69 years (21.0\%, Figure 1D). The prevalence and standardized prevalence of NAFLD in RA patients among different age groups shared similar characteristics to MAFLD (Figure 1B, 1D). 
The active RA patients had a higher prevalence of MAFLD and NAFLD than those in remission (MAFLD: 23.1\% vs. 12.0\%, $p=$ 0.031; NAFLD: $22.1 \%$ vs. $10.7 \%, p=0.023$, Figure 1E). However, there was no significant difference of MAFLD or NAFLD prevalence among RA patients with different disease duration (Figure 1F).

\section{Clinical and metabolic characteristics in RA patients with MAFLD or NAFLD}

RA patients with MAFLD or NAFLD were older than those without (MAFLD: $54.4 \pm 9.5$ years vs. $51.1 \pm 13.2$ years, $p=0.027$; NAFLD: $54.2 \pm 9.7$ years vs. $51.2 \pm 13.2$ years, $p=0.043$, respectively, Table 1), but no significant differences of other RA disease characteristics were observed between 2 groups. As expected, RA patients with MAFLD or NAFLD had a significantly higher prevalence of metabolic abnormalities (Supplementary Table S1). In turn, RA patients with metabolic abnormalities, including T2DM, elevated TG, overweight/obesity, elevated WC, elevated HOMA-IR, and elevated BP, had a significantly higher prevalence of MAFLD and NAFLD (Supplementary Figure S1). Notably, RA patients with T2DM had the highest prevalence of MAFLD (43.0\%) and NAFLD (39.2\%), followed by RA patients with elevated TG (MAFLD: 42.9\%; NAFLD: 39.7\%), overweight/obesity (MAFLD: $41.1 \%$; NAFLD: $38.1 \%$ ).

\section{Liver biochemistry and fibrosis indices in RA patients with MAFLD or NAFLD}

Both RA patients with MAFLD and those with NAFLD had slightly elevated ALT, ULN < ALT < 2 ULN (MAFLD: $11.8 \%$ vs. $4.5 \%$; NAFLD: $12.4 \%$ vs. $4.4 \%$, both $p<0.05$, Table 2). In addition, RA patients with MAFLD had a higher liver fibrosis score (NFS: median -1.56 vs. -2.19; Forns index: median 5.19 vs. 4.85, respectively), and a higher percentage of advanced fibrosis (NFS: $36.4 \%$ vs. 15.9\%; Forns index: $33.6 \%$ vs. 22.6\%). Similar findings were found for RA patients with NAFLD (liver fibrosis score: NFS: median -1.62 vs. -2.17 and Forns index: median 5.02 vs. 4.86, respectively; advanced fibrosis: only in NFS: $36.2 \%$ vs. $16.2 \%$, all $p<0.05$, Table 2 ).

\section{Characteristics of RA patients according to the MAFLD subtypes}

According to the criteria of MAFLD, RA patients with MAFLD were divided into 3 subgroups: overweight/obesity $(n=48$, $43.6 \%)$, T2DM $(n=34,30.9 \%)$ and lean/ normal weight $(n=28,25.5 \%)$. Among the three subtypes of MAFLD, RA patients with T2DM MAFLD were older than those with overweight/obesity MAFLD (58.9 \pm 7.6 years vs. $51.9 \pm 9.9$ years, $p=0.003)$, but there were no significant differences in other RA disease characteristics (Supplementary Table S2). There were no significant differences in the prevalence of metabolic abnormalities except for the highest prevalence of elevated WC in RA patients with overweight/obesity MAFLD and highest prevalence of prediabetes in RA patients with lean/normal weight MAFLD (Supplementary Figure S2).

The comparisons of liver biochemistry and fibrosis indices among RA patients with three subtypes of MAFLD were shown in Supplementary Table S3. There were no significant differences in liver biochemistry among RA patients with three subtypes of MAFLD. While RA patients with T2DM MAFLD showed the highest NFS (median -0.64 vs. -2.36 vs. $-1.84, p<0.001$ ) and

Forns index (median 5.62 vs. 4.61 vs. 4.84, $p=0.008$ ), and the highest proportion of advanced fibrosis defined by NFS (58.8\% vs. $21.4 \%$ vs. $29.2 \%, p=0.004)$ compared with those lean/normal weight and overweight/obesity MAFLD subgroups. Furthermore, RA patients with T2DM MAFLD had a significantly higher FIB-4 level (median 0.95 vs. 0.72, $p=0.036$ ) and advanced fibrosis ratio defined by FIB-4 $(29.4 \%$ vs. $6.3 \%, p=0.011)$ and by Forns index $(52.9 \%$ vs. $22.9 \%, p=0.015)$ compared with those with overweight/obesity MAFLD.

\section{CVD events and 10-year CVD risk in RA patients with MAFLD or NAFLD}


In this RA cohort, 56 (10.9\%) patients concomitated with CVD events, including ischemic stroke (4.5\%), heart failure (3.7\%), myocardial infarction (2.3\%), angina pectoris (1.4\%) and peripheral arterial disease (0.6\%). Compared with those without, RA patients with MAFLD or NAFLD had a higher incidence of CVD events (MAFLD: 17.3\% vs. 9.2\%; NAFLD: 18.1\% vs. 9.1\%), especially higher rates of myocardial infarction (MAFLD: $5.5 \%$ vs. 1.5\%; NAFLD: $5.7 \%$ vs. $1.5 \%$ ) and angina pectoris (MAFLD: $3.6 \%$ vs. $0.7 \%$; NAFLD: $3.8 \%$ vs. $0.7 \%$, all $p<0.05$, Table 3). RA patients with MAFLD or NAFLD also had significantly higher 10-year CVD risk scores including China-PAR, SCORE and FRS (Table 3). The median China-PAR were significantly higher in RA patients with MAFLD or NAFLD (MAFLD: $3.7 \%$ vs. $2.1 \%$; NAFLD: $3.7 \%$ vs. $2.1 \%$, respectively, all $p<0.001$ ).

A total of 166 (32.4\%) RA patients had a high 10-year CVD risk. RA patients with MAFLD or NAFLD had higher proportion of high 10-year CVD risk than those without (MAFLD: 55.5\% vs. 26.1\%; NAFLD: 55.2\% vs. 26.5\%, respectively, all $p<0.001$, Table 3).

\section{Factors associated with CVD events and high 10-year CVD risk in RA patients}

As shown in Table 4, univariate logistic regression analysis showed that MAFLD or NAFLD was associated with a higher risk of CVD events in RA patients (MAFLD: OR $=2.065,95 \% \mathrm{Cl} 1.135-3.759, p=0.018$; NAFLD: OR $=2.215,95 \% \mathrm{Cl} 1.215-4.040, p=$ 0.009 , respectively). And the associations remained after adjustment for the potential confounders (MAFLD: adjusted OR $[\mathrm{AOR}]=2.303,95 \% \mathrm{Cl} 1.197-4.429, p=0.012$; NAFLD: AOR $=2.478,95 \% \mathrm{Cl} 1.185-4.779, p=0.007$, respectively). Further analysis stratified by the MAFLD subtypes showed a significant association of T2DM MAFLD with risk of CVD events $(\mathrm{OR}=3.044,95 \% \mathrm{Cl} 1.286-7.204, p=0.011)$.

Moreover, MAFLD or NAFLD was associated with the high 10-year CVD risk for patients with RA in the unadjusted analysis (MAFLD: OR $=3.533,95 \% \mathrm{Cl} 2.283-5.469, p<0.001$; NAFLD: OR $=3.428,95 \% \mathrm{Cl} 2.201-5.339, p<0.001$, respectively). After adjustment for the potential confounders, these associations remained (MAFLD: AOR $=3.184,95 \% \mathrm{Cl} 1.777-5.705, p<0.001$; NAFLD: $A O R=2.870,95 \% \mathrm{Cl} 1.597-5.156, p<0.001$, respectively).

To compare the performance of MAFLD and NAFLD in identifying CVD events and high 10-years CVD risk in RA patients, the $\mathrm{NRI}$ and IDI were performed to evaluate the improvement of diagnostic accuracy. The NRI and IDI was $-0.011(95 \% \mathrm{Cl}-0.025-$ 0.003 ) and -0.002 (95\% Cl -0.007-0.002) for CVD events, and 0.012 (95\% Cl-0.014-0.038) and 0.005 (95\% Cl -0.003-0.013, all $p>0.05$, Supplementary Table S4) for high 10-year CVD risk, respectively. Compared with NAFLD, the MAFLD criteria had no further improvement (either measured by NRI or IDI) in identifying CVD events and high 10-years CVD risk.

\section{Discussion}

Our research for the first reports that the prevalence of MAFLD in RA patients is $21.4 \%$. Besides a higher risk of advanced fibrosis, both MAFLD and NAFLD are related to an increased hazard (2 3 folds) of CVD events and high 10-year CVD risk. However, new nomenclature from NAFLD to MAFLD identifies no additional CVD risk in RA patients. These findings impose an emphasis on the detection and management of MAFLD or NAFLD in patients with RA.

A recent meta-analysis in 2020 reported the total prevalence of NAFLD in Mainland China is $29.9 \%$, which was similar to that in Western countries (23.7\%-31.8\%) [23,24]. And the epidemiological data of MAFLD prevalence was $30 \%-40 \%$ in general population in Asia [25]. Although the definition of MAFLD allowed the inclusion of patients with over alcohol intake and concomitant liver diseases, dispute still remained on whether more patients were identified by using the MAFLD definition. In a Korea Nationwide Cohort Study of 9.58 million middle-aged adults, the prevalence of fatty liver disease (FLD) was higher by using the definition of MAFLD than NAFLD (37.3\% vs. 28.0\%) [26]. A population-based study in the northern China also showed a higher prevalence of MAFLD than NAFLD (32.7\% vs. 27.3\%) [27]. However, other researchers showed an identical prevalence between MAFLD and NAFLD (31.2\% vs. 33.2\%) by Lin et al. [28] and 25.9\% vs. 25.7\% by Wong et al. [29]. In our cohort, the prevalence of NAFLD was $20.5 \%$, which was consistent with that in Korean (21.1\%) and in Italian (25.0\%) RA

Page 6/19 
population [30,31]. To our best knowledge, no studies have investigated the epidemiological data of MAFLD in RA patients yet. In this study, the prevalence of MAFLD was $21.4 \%$, which indicated the new definition of MAFLD shared a similar prevalence to NAFLD in RA patients.

Reports from general population have demonstrated that individuals with MAFLD have a higher level of liver parameters (ALT, AST, ALP and GGT) and more proportion of abnormal ALT than those without [26, 27]. MAFLD patients showed a higher liver fibrosis score and a higher ratio of advanced fibrosis than the general population [28,29]. Moreover, the sensitivity for detecting advanced fibrosis was higher for MAFLD than NAFLD (93.9\% vs. 73.0\%), in which the MAFLD definition better identifies advanced fibrosis in general population [32]. In our study, RA patients with MAFLD or NAFLD showed slightly elevated ALT, higher liver fibrosis scores and advanced fibrosis. As recommended by 2021 ACR Guideline for RA treatment [33], the clinicians should pay attention to NAFLD (or MAFLD) and dynamically monitor liver enzymes, liver function tests and liver fibrosis when using methotrexate and other hepatic toxic medicines.

CVD is the primary reason for deaths among RA patients. Previous research reported RA patients were more likely to have a history of myocardial infarction (3.1\% vs. $2.6 \%)$, heart failure $(1.6 \%$ vs. $1.0 \%)$ or stroke $(3.9 \%$ vs. $2.7 \%)$ at diagnosis, and a higher number of CVD events occurred (events per 1000 person-years $10.6 \mathrm{vs}$. 8.1) in the first 5 years than controls [34]. In our RA cohort, 56 (10.9\%) patients concomitated with CVD events, including ischemic stroke (4.5\%), heart failure (3.7\%), myocardial infarction (2.3\%), angina pectoris (1.4\%), and peripheral arterial disease (0.6\%). The prevalence of CVD events in our study $(10.9 \%, 56 / 513)$ was similar to a large-scale multicenter survey of 21 hospitals in China $(12.7 \%, 256 / 2013)$ [35]. A comprehensive meta-analysis demonstrated that NAFLD conferred an OR of 1.64 for fatal and/or non-fatal CVD events [36]. Several recent studies also showed that MAFLD was related to an increased hazard of CVD than those without (1.43 folds) by Lee et al [26] and 1.89 folds by Yoneda et al [37]. These data indicated that both NAFLD and MAFLD were independent risk factors for CVD in general population. There are multiple underlying mechanisms by which FLD increases the risk of CVD, such as systemic inflammation and oxidative stress, hepatic insulin resistance, platelet activation, endothelial dysfunction, et al [7]. In the present study, we firstly reported that RA patients with MAFLD or NAFLD was associated with an increased CVD risk, including CVD events (MAFLD: AOR = 2.303; NAFLD: AOR = 2.478) and high 10-year CVD risk (MAFLD: AOR = 3.184; NAFLD: $A O R=2.870$ ). According to these data, all RA patients with MAFLD or NAFLD should undergo careful CVD surveillance as recommended by the American Association for the Study for Liver Diseases (AASLD) guidelines for all patients with MAFLD or NAFLD, either by Framingham risk score, SCORE or by other risk charts for CVD risk assessments $[38,39]$.

Recently, a large-scale matched cohort research including 17.7 million participants showed that NAFLD was not significantly related to stroke or acute myocardial infarction risk after adjustment for established CVD risk factors [40]. In this concern, the alteration from NAFLD to MAFLD was proposed for identifying more patients at an increased risk of CVD in the general population $[26,41]$. Our research for the first reported that both MAFLD and NAFLD was associated with an increased CVD risk. Compared with NAFLD, the MAFLD criteria had no further improvement in either NRI or IDI, which indicated that the alteration from NAFLD to MAFLD criteria didn't recognize additional CVD risks in RA patients. On the one hand, as an inflammatory disease, RA is an important risk factor for CVD [3]. Meanwhile, systemic inflammation is also implicated in the progression of liver fibrosis, which was identical between the NAFLD and MAFLD [6,7]. On the other hand, the presence of metabolic dysfunction is the main reason responsible for the superiority of MAFLD over NAFLD to predict CVD risk [26,41]. However, in this study, the rate of overweight/obesity, a key metabolic trait of MAFLD, in Chinese RA patients was $32.7 \%$, which was lower than that in Chinese general adults (50.7\%) [42]. All these data indicated that whether it is appropriate to rename NAFLD to MAFLD in RA patients warrants further studies.

T2DM and IR are the most important risk factors for NAFLD or MAFLD development, which strongly predict adverse clinical outcomes such as advanced hepatic fibrosis and mortality $[43,44]$. In turn, fat accumulation in the liver causes hepatic IR, boosts increased glucose production in the liver, and in turn acts as a further stimulus for increasing whole-body IR [45] and CVD risks [46]. MAFLD (defined by hepatic steatosis index > 36) was present in 76.3\% of the 78,895 T2DM patients in Italian specialist care [47]. Diabetes MAFLD had more significant fibrosis than overweight, obese, lean/normal weight MAFLD

Page 7/19 
(18.9\% vs. $1.3 \%$ vs. $5.5 \%$ vs. $6.4 \%$ ) [47]. In the present study, the prevalence of T2DM in RA patients with MAFLD was $30.9 \%$, and RA patients with T2DM had a higher prevalence of MAFLD than non-T2DM RA patients (43.0\% vs. 17.5\%), similar to that in the general population (47.2\% vs. 25.9\%) [48]. In RA patients with T2DM MAFLD, both fibrosis score and advanced fibrosis ratio were higher than the other two subtypes of MAFLD (defined by NFS: T2DM 58.8\%, overweight/obesity $29.2 \%$, lean/normal weight 21.4\%). RA patients with T2DM MAFLD were related to a raised hazard of CVD events $(O R=3.044)$. These data implied that monitoring of MAFLD and CVD risk should be emphasized in RA patients especially with T2DM.

There were several potential limitations in this study. First, ultrasound, rather than liver biopsy, was used to identify hepatic steatosis. However, one qualified meta-analysis showed a high sensitivity and specificity in the detection of moderate-severe hepatic steatosis by ultrasound [49]. Second, CRP rather than hs-CRP was regarded as one of the indicators of metabolic abnormalities in lean/normal weight MAFLD definition in our study. However, the remaining 3 patients who were not diagnosed with MAFLD because had neither T2DM, overweight/obesity nor any other metabolic disorders than CRP. Thus, the prevalence of MAFLD was not affected by CRP in our study. Third, as for the nature of any observational studies, we cannot exclude the possibility of residual confounding completely despite our careful adjustment for the potential risk factors.

\section{Conclusions}

In conclusion, MAFLD and NAFLD are common comorbidities in RA patients and both are associated with increased CVD risk. However, new nomenclature from NAFLD to MAFLD identifies no additional CVD risks. These data imply an importance of MAFLD or NAFLD detection and dynamic monitoring in RA patients especially with T2DM or overweight/obesity. All RA patients with MAFLD or NAFLD should undergo careful cardiovascular surveillance and primary prevention, and treatments to modify MAFLD or NAFLD also need consideration in the management of RA.

\section{Abbreviations}

NAFLD: Non-alcoholic fatty liver disease; MAFLD: Metabolic dysfunction-associated fatty liver disease; CVD: Cardiovascular disease; RA: Rheumatoid arthritis; T2DM: Type 2 diabetes mellitus; ALT: Alanine aminotransferase; AST: Aspartate aminotransferase; GGT: y-glutamyl transferase; ALP: Alkaline phosphatase; TBIL: Total bilirubin; ALB: Albumin; FPG: Fasting plasma glucose; HbA1c: Glycated hemoglobin; FINS: Fasting insulin; TC: Total cholesterol; TG: Triglyceride; HDL-C: Highdensity lipoprotein cholesterol; LDL-C: Low-density lipoprotein cholesterol; LFTs: Liver function tests; ULN: Upper limit of normal; HOMA-IR: Homeostasis model assessment of insulin resistance; IR: Insulin resistance; FLI: Fatty liver index; FIB-4: Fibrosis-4 index; NFS: NAFLD fibrosis score; BMI: Body mass index; BP: Blood pressure; WC: Waist circumference; hs-CRP: High-sensitivity C-reactive protein; ASCVD: Atherosclerotic cardiovascular disease; China-PAR: Prediction for Atherosclerotic Cardiovascular Disease Risk in China; SCORE: Systemic Coronary Risk Evaluation; FRS: Framingham Risk Score; OR: Odds ratio; NRI: Net reclassification index; IDI: Integrated discrimination improvement; AOR: Adjusted OR; AASLD: American Association for the Study for Liver Diseases; RF: Rheumatoid factor; ACPA: Anti-cyclic citrullinated peptide antibody; 28TJC: 28-joint tender joint counts; 28SJC: 28-joint swollen joint counts; PtGA: Patient global assessment of disease activity; PrGA: Provider global assessment of disease activity; Pain VAS: Pain visual analogue scale; ESR: Erythrocyte sedimentation rate; CRP: C reactive protein; CDAl: Clinical disease activity index; HAQ-DI: Health assessment questionnaire disability index; mTSS: Modified total Sharp score; JE: Joint erosion; JSN: Joint space narrowing; csDMARDs: Conventional synthetic disease-modifying anti-rheumatic drugs.

\section{Declarations}

\section{Acknowledgements}

We thank all subjects and medical staff who generously contributed to this study. 


\section{Author Contributions}

YWZ and QHL contributed equally to this work, including conceiving and designing the study, reading and analyzing documents, performing statistical analysis, and drafting the manuscript. JWG participated in analyzing documents and drafting the paper. JP and LFC participated in clinical assessment. JDM and JZL carried out the radiographic assessment. YQM and XPZ participated in data collection. Corresponding authors PML and LD conceived and participated in its design, read and analyzed documents, and edited the paper. All authors read and approved the final manuscript.

\section{Funding}

This study was supported by National Natural Science Foundation of China (No. 81971527, 82171780 and 82101892), Science and Technology Program of Guangzhou (No. 202102010188 and 201904010088), and Guangdong Basic and Applied Basic Research Foundation (No. 2019A1515011928 and 2020A1515110061).

\section{Availability of data}

The datasets used during the current study are available from the corresponding author Lie Dai on reasonable request.

\section{Ethics approval and consent to participate}

Ethical approval mandatory for this study was obtained from Ethics Committee at Sun Yat-sen Memorial Hospital (SYSECKY-KS-2020-208) along with an informed consent from each patient.

\section{Consent for publication}

This study has obtained consent to publish from the participants to report individual patient data. Details that might disclose the identity of the participants under study have been omitted.

\section{Competing interests}

The authors declare that they have no known competing financial interests or personal relationships that could have appeared to influence the work reported in this paper.

\section{References}

1. Figus FA, Piga M, Azzolin I, et al. Rheumatoid arthritis: Extra-articular manifestations and comorbidities. Autoimmun Rev. 2021;20(4):102776.

2. Crowson CS, Liao KP, Davis JM 3rd, et al. Rheumatoid arthritis and cardiovascular disease. Am Heart J. 2013;166(4):622628.e1.

3. Semb AG, Ikdahl E, Wibetoe G, et al. Atherosclerotic cardiovascular disease prevention in rheumatoid arthritis. Nat Rev Rheumatol. 2020;16(7):361-379.

4. Aviña-Zubieta JA, Choi HK, Sadatsafavi M, et al. Risk of cardiovascular mortality in patients with rheumatoid arthritis: a meta-analysis of observational studies. Arthritis Rheum. 2008;59(12):1690-1697.

5. Radovanović-Dinić B, Tešić-Rajković S, Zivkovic V, et al. Clinical connection between rheumatoid arthritis and liver damage. Rheumatol Int. 2018;38(5):715-724. 
6. Powell EE, Wong VW, Rinella M. Non-alcoholic fatty liver disease. Lancet. 2021;397(10290):2212-2224.

7. Targher G, Byrne CD, Tilg H. NAFLD and increased risk of cardiovascular disease: clinical associations, pathophysiological mechanisms and pharmacological implications. Gut. 2020;69(9):1691-1705.

8. Eslam M, Newsome PN, Sarin SK, et al. A new definition for metabolic dysfunction-associated fatty liver disease: an international expert consensus statement. J Hepatol. 2020;73(1):202-209.

9. Wong VW, Lazarus JV. Prognosis of MAFLD vs. NAFLD and implications for a nomenclature change. J Hepatol. 2021;75(6):1267-1270.

10. Lin JZ, Liang JJ, Ma JD, et al. Myopenia is associated with joint damage in rheumatoid arthritis: a cross-sectional study. J Cachexia Sarcopenia Muscle. 2019, 10(2):355-367.

11. Lin JZ, Liu Y, Ma JD, et al. Reduced skeletal muscle independently predicts 1-year aggravated joint destruction in patients with rheumatoid arthritis. Ther Adv Musculoskelet Dis. 2020;12:1759720X20946220.

12. Lin JZ, Chen CT, Ma JD, et al. Neglected extra-articular manifestations in rheumatoid arthritis patients with normal body mass index: reduced skeletal muscle overlapping overfat. Ther Adv Chronic Dis. 2020;11:2040622320975241.

13. Ma JD, Chen CT, Lin JZ, et al. Muscle wasting, a neglected complication associated with physical dysfunction in elderly patients with rheumatoid arthritis: a cross-sectional observational study. Scand J Rheumatol. 2021;50(4):280-289.

14. Aletaha D, Neogi T, Silman AJ, et al. 2010 rheumatoid arthritis classification criteria: an American College of Rheumatology/European League Against Rheumatism collaborative initiative. Ann Rheum Dis. 2010, 69(9):1580-1588.

15. Newsome PN, Cramb R, Davison SM, et al. Guidelines on the management of abnormal liver blood tests. Gut. 2018;67(1):6-19.

16. Wallace TM, Levy JC, Matthews DR. Use and abuse of HOMA modeling. Diabetes Care. 2004;27:1487-1495.

17. Rasmussen DN, Thiele M, Johansen S, et al. Prognostic performance of 7 biomarkers compared to liver biopsy in early alcohol-related liver disease. J Hepatol. 2021;75(5):1017-1025.

18. Yang X, Li J, Hu D, et al. Predicting the 10-Year Risks of Atherosclerotic Cardiovascular Disease in Chinese Population: The China-PAR Project (Prediction for ASCVD Risk in China). Circulation. 2016;134(19):1430-1440.

19. Piepoli MF, Hoes AW, Agewall S, et al. 2016 European Guidelines on cardiovascular disease prevention in clinical practice: The Sixth Joint Task Force of the European Society of Cardiology and Other Societies on Cardiovascular Disease Prevention in Clinical Practice (constituted by representatives of 10 societies and by invited experts) Developed with the special contribution of the European Association for Cardiovascular Prevention \& Rehabilitation (EACPR). Eur Heart J. 2016;37(29):2315-2381.

20. D'Agostino RB Sr, Vasan RS, Pencina MJ, et al. General cardiovascular risk profile for use in primary care: the Framingham Heart Study. Circulation. 2008;117(6):743-753.

21. Chinese Society of Cardiology of Chinese Medical Association; Cardiovascular Disease Prevention and Rehabilitation Committee of Chinese Association of Rehabilitation Medicine; Cardiovascular Disease Committee of Chinese Association of Gerontology and Geriatrics; Thrombosis Prevention and Treatment Committee of Chinese Medical Doctor Association. Zhonghua Xin Xue Guan Bing Za Zhi. 2020;48(12):1000-1038.

22. National Bureau of Statistics of China. 2020 China Statistical Yearbook. http://www.stats.gov.cn/tjsj/ndsj/2020/indexeh.htm.

23. Wu Y, Zheng Q, Zou B, et al. The epidemiology of NAFLD in Mainland China with analysis by adjusted gross regional domestic product: a meta-analysis. Hepatol Int. 2020;14(2):259-269.

24. Younossi ZM, Koenig AB, Abdelatif D, et al. Global epidemiology of nonalcoholic fatty liver disease-Meta-analytic assessment of prevalence, incidence, and outcomes. Hepatology. 2016;64(1):73-84.

25. Hui Lim GE, Tang A, Ng CH, et al. An Observational Data Meta-Analysis on the Differences in Prevalence and Risk Factors Between MAFLD vs NAFLD. Clin Gastroenterol Hepatol. 2021;S1542-3565(21)01276-3. 
26. Lee H, Lee YH, Kim SU, et al. Metabolic dysfunction-associated fatty liver disease and incident cardiovascular disease risk: a nationwide cohort study. Clin Gastroenterol Hepatol. 2021;19(10):2138-2147.e10.

27. Wang X, Wu S, Yuan X, et al. Metabolic dysfunction-associated fatty liver disease and mortality among Chinese adults: a prospective cohort study. J Clin Endocrinol Metab. 2021;dgab644.

28. Lin S, Huang J, Wang M, et al. Comparison of MAFLD and NAFLD diagnostic criteria in real world. Liver Int. 2020;40(9):2082-2089.

29. Wong VW, Wong GL, Woo J, et al. Impact of the new definition of metabolic associated fatty liver disease on the epidemiology of the disease. Clin Gastroenterol Hepatol. 2021;19(10):2161-2171.e5.

30. Ursini F, Russo E, Mauro D, et al. Complement C3 and fatty liver disease in Rheumatoid arthritis patients: a crosssectional study. Eur J Clin Invest. 2017;47(10):728-735.

31. Choi Y, Lee $\mathrm{CH}, \mathrm{Kim} \mathrm{IH}$, et al. Methotrexate use does not increase the prevalence of hepatic steatosis: a real-world retrospective nested case-control study. Clin Rheumatol. 2021;40(5):2037-2045.

32. Yamamura S, Eslam M, Kawaguchi T, et al. MAFLD identifies patients with significant hepatic fibrosis better than NAFLD. Liver Int. 2020;40(12):3018-3030.

33. Fraenkel L, Bathon JM, England BR, et al. 2021 American College of Rheumatology Guideline for the Treatment of Rheumatoid Arthritis. Arthritis Care Res (Hoboken). 2021;73(7):924-939.

34. Nikiphorou E, de Lusignan S, Mallen CD, et al. Cardiovascular risk factors and outcomes in early rheumatoid arthritis: a population-based study. Heart. 2020;106(20):1566-1572.

35. Li C, Wang XR, Ji HJ, et al. Cardiovascular disease in rheumatoid arthritis: medications and risk factors in China. Clin Rheumatol. 2017;36(5):1023-1029.

36. Targher G, Byrne CD, Lonardo A, et al. Non-alcoholic fatty liver disease and risk of incident cardiovascular disease: a meta-analysis. J Hepatol. 2016;65(3):589-600.

37. Yoneda M, Yamamoto T, Honda Y, et al. Risk of cardiovascular disease in patients with fatty liver disease as defined from the metabolic dysfunction associated fatty liver disease or nonalcoholic fatty liver disease point of view: a retrospective nationwide claims database study in Japan. J Gastroenterol. 2021;56(11):1022-1032.

38. European Association for the Study of the Liver (EASL); European Association for the Study of Diabetes (EASD); European Association for the Study of Obesity (EASO). EASL-EASD-EASO Clinical Practice Guidelines for the management of non-alcoholic fatty liver disease. Diabetologia. 2016;59(6):1121-1140.

39. Chalasani N, Younossi Z, Lavine JE, et al. The diagnosis and management of nonalcoholic fatty liver disease: Practice guidance from the American Association for the Study of Liver Diseases. Hepatology. 2018;67(1):328-357.

40. Alexander M, Loomis AK, van der Lei J, et al. Non-alcoholic fatty liver disease and risk of incident acute myocardial infarction and stroke: findings from matched cohort study of 18 million European adults. BMJ. 2019;367:15367.

41. Tsutsumi T, Eslam M, Kawaguchi T, et al. MAFLD better predicts the progression of atherosclerotic cardiovascular risk than NAFLD: Generalized estimating equation approach. Hepatol Res. 2021;51(11):1115-1128.

42. Pan XF, Wang L, Pan A. Epidemiology and determinants of obesity in China. Lancet Diabetes Endocrinol. 2021;9(6):373392.

43. Younossi ZM, Golabi P, de Avila L, et al. The global epidemiology of NAFLD and NASH in patients with type 2 diabetes: a systematic review and meta-analysis. J Hepatol. 2019;71(4):793-801.

44. Lim S, Kim JW, Targher G. Links between metabolic syndrome and metabolic dysfunction-associated fatty liver disease. Trends Endocrinol Metab. 2021;32(7):500-514.

45. Davis TME. Diabetes and metabolic dysfunction-associated fatty liver disease. Metabolism. 2021;123:154868.

46. Morieri ML, Vitturi N, Avogaro A, et al. Prevalence of hepatic steatosis in patients with type 2 diabetes and response to glucose-lowering treatments. A multicenter retrospective study in Italian specialist care. J Endocrinol Invest. 2021;44(9):1879-1889.

Page $11 / 19$ 
47. Sohn W, Kwon HJ, Chang Y, et al. Liver fibrosis in Asians with metabolic dysfunction-associated fatty liver disease. Clin Gastroenterol Hepatol. 2021;S1542-3565(21)00706-0.

48. Fan J, Luo S, Ye Y, et al. Prevalence and risk factors of metabolic associated fatty liver disease in the contemporary South China population. Nutr Metab (Lond). 2021;18(1):82.

49. Hernaez R, Lazo M, Bonekamp S, et al. Diagnostic accuracy and reliability of ultrasonography for the detection of fatty liver: a meta-analysis. Hepatology. 2011;54(3):1082-1090.

\section{Tables}

\section{Table 1}

Demographic and clinical characteristics of RA patients with MAFLD or NAFLD 


\begin{tabular}{|c|c|c|c|c|c|c|c|}
\hline Characteristics & $\begin{array}{l}\text { RA patients } \\
(n=513)\end{array}$ & $\begin{array}{l}\text { No-MAFLD } \\
(n=403)\end{array}$ & $\begin{array}{l}\text { MAFLD } \\
(n=110)\end{array}$ & $P *$ & $\begin{array}{l}\text { No-NAFLD } \\
(n=408)\end{array}$ & $\begin{array}{l}\text { NAFLD } \\
(n=105)\end{array}$ & $P \#$ \\
\hline Age, years & $51.8 \pm 12.6$ & $51.1 \pm 13.2$ & $54.4 \pm 9.5$ & 0.027 & $51.2 \pm 13.2$ & $54.2 \pm 9.7$ & 0.043 \\
\hline Female, $n(\%)$ & $402(78.4)$ & 317 (78.7) & $85(77.3)$ & 0.754 & $320(78.4)$ & $82(78.1)$ & 0.941 \\
\hline $\begin{array}{l}\text { Active smoking, } n \\
(\%)\end{array}$ & $90(17.5)$ & 68 (16.9) & $22(20.0)$ & 0.445 & 69 (16.9) & $21(20.0)$ & 0.458 \\
\hline $\begin{array}{l}\text { Disease duration, } \\
\text { months }\end{array}$ & $60(21,120)$ & $57(20,120)$ & $60(24,132)$ & 0.415 & $55(20,120)$ & $60(24,132)$ & 0.362 \\
\hline Positive RF, $n(\%)$ & $366(71.3)$ & $287(71.2)$ & $79(71.8)$ & 0.901 & $291(71.3)$ & 75 (71.4) & 0.983 \\
\hline Positive ACPA, $n(\%)$ & $370(72.1)$ & $289(71.7)$ & 81 (73.6区) & 0.690 & $293(71.8)$ & 77 (73.3) & 0.757 \\
\hline \multicolumn{8}{|l|}{$\begin{array}{l}\text { Disease activity } \\
\text { indicators }\end{array}$} \\
\hline 28TJC & $4(1,9)$ & $4(1,10)$ & $3(1,8)$ & 0.206 & $4(1,9)$ & $3(1,8)$ & 0.297 \\
\hline 28SJC & $2(0,7)$ & $2(0,7)$ & $1(0,6)$ & 0.209 & $2(0,7)$ & $1(0,6)$ & 0.482 \\
\hline PtGA, cm & $4(2,6)$ & $4(2,6)$ & $4(2,6)$ & 0.524 & $4(2,6)$ & $4(2,6)$ & 0.437 \\
\hline PrGA, cm & $4(2,6)$ & $4(2,6)$ & $4(2,6)$ & 0.341 & $4(2,6)$ & $4(2,6)$ & 0.224 \\
\hline Pain VAS, cm & $4(2,5)$ & $4(2,5)$ & $4(2,5)$ & 0.690 & $4(2,5)$ & $4(2,5)$ & 0.497 \\
\hline $\mathrm{ESR}, \mathrm{mm} / \mathrm{h}$ & $38(20,70)$ & $40(20,70)$ & $34(20,63)$ & 0.282 & $39(20,70)$ & $35(21,65)$ & 0.642 \\
\hline $\mathrm{CRP}, \mathrm{mg} / \mathrm{L}$ & $\begin{array}{l}7.6 \\
(3.3,27.6)\end{array}$ & $\begin{array}{l}8.3 \\
(3.3,30.6)\end{array}$ & $\begin{array}{l}6.4 \\
(3.3,21.4)\end{array}$ & 0.628 & $\begin{array}{l}8.1 \\
(3.3,30.5)\end{array}$ & $\begin{array}{l}7.2 \\
(3.3,21.6)\end{array}$ & 0.869 \\
\hline CDAI & $14(6,27)$ & $14(6,28)$ & $14(6,26)$ & 0.725 & $14(6,28)$ & $15(6,27)$ & 0.987 \\
\hline \multicolumn{8}{|l|}{ Functional indicator } \\
\hline HAQ-DI & $\begin{array}{l}0.50 \\
(0.00,1.13)\end{array}$ & $\begin{array}{l}0.38 \\
(0.00,1.25)\end{array}$ & $\begin{array}{l}0.62 \\
(0.13,1.13)\end{array}$ & 0.226 & $\begin{array}{l}0.38 \\
(0.00,1.25)\end{array}$ & $\begin{array}{l}0.63 \\
(0.13,1.13)\end{array}$ & 0.192 \\
\hline \multicolumn{8}{|l|}{$\begin{array}{l}\text { Radiographic } \\
\text { indicators }\end{array}$} \\
\hline mTSS & $12(4,40)$ & $10(1,34)$ & $9(2,33)$ & 0.657 & $10(1,34)$ & $9(2,30)$ & 0.698 \\
\hline JSN subscore & $4(0,18)$ & $5(0,19)$ & $2(0,17)$ & 0.093 & $5(0,19)$ & $2(0,17)$ & 0.177 \\
\hline JE subscore & $8(3,22)$ & $8(2,22)$ & $8(3,23)$ & 0.859 & $8(2,22)$ & $8(3,21)$ & 0.910 \\
\hline \multicolumn{8}{|l|}{$\begin{array}{l}\text { Previous } \\
\text { medications }\end{array}$} \\
\hline $\begin{array}{l}\text { Treatment naïve }{ }^{\triangle}, n \\
(\%)\end{array}$ & $141(27.5)$ & $107(26.6)$ & 34 (30.9) & 0.364 & $109(26.7)$ & $32(30.5)$ & 0.441 \\
\hline Glucocorticoid, $n(\%)$ & $262(51.1)$ & $202(50.1)$ & $60(54.5)$ & 0.411 & $204(50.0)$ & $58(55.2)$ & 0.338 \\
\hline csDMARDs, $n(\%)$ & $322(62.8)$ & $258(64.0)$ & $64(58.2)$ & 0.262 & $261(64.0)$ & $61(58.1)$ & 0.267 \\
\hline $\begin{array}{l}\text { Biologic agents, } n \\
(\%)\end{array}$ & $42(8.2)$ & $34(8.4)$ & $8(7.3)$ & 0.693 & $33(8.1)$ & $9(8.6)$ & 0.872 \\
\hline
\end{tabular}

$\triangle$ Treatment naive, without previous corticosteroids or DMARDs treatment for six months before recruited. 
RF, rheumatoid factor; ACPA, anti-cyclic citrullinated peptide antibody; 28TJC, 28-joint tender joint counts; 28SJC, 28-joint swollen joint counts; PtGA, patient global assessment of disease activity; PrGA, provider global assessment of disease activity; Pain VAS, pain visual analogue scale; ESR, erythrocyte sedimentation rate; CRP, C reactive protein; CDAI, clinical disease activity index; HAQ-DI, health assessment questionnaire disability index; mTSS, modified total Sharp score; JE, joint erosion; JSN, joint space narrowing; csDMARDs, conventional synthetic disease-modifying anti-rheumatic drugs

* Compared between RA patients with or without MAFLD

\# Compared between RA patients with or without NAFLD

Table 2

Comparisons of liver biochemistry and fibrosis indices between RA patients with and without MAFLD or NAFLD 


\begin{tabular}{|c|c|c|c|c|c|c|c|}
\hline Characteristics & $\begin{array}{l}\text { RA patients } \\
(n=513)\end{array}$ & $\begin{array}{l}\text { No-MAFLD } \\
(n=403)\end{array}$ & $\begin{array}{l}\text { MAFLD } \\
(n=110)\end{array}$ & $P^{*}$ & $\begin{array}{l}\text { No-NAFLD } \\
(n=408)\end{array}$ & $\begin{array}{l}\text { NAFLD } \\
(n=105)\end{array}$ & $P \#$ \\
\hline \multicolumn{8}{|l|}{ liver biochemistry } \\
\hline$A L T, U I / L$ & $16(10,23)$ & $15(10,23)$ & $17(11,27)$ & 0.085 & $15(10,23)$ & $17(11,27)$ & 0.066 \\
\hline $\begin{array}{l}\text { ULN < } \mathrm{ALT}<2 \mathrm{ULN}, \mathrm{n} \\
(\%)\end{array}$ & $31(6.0)$ & $18(4.5)$ & $13(11.8)$ & 0.004 & $18(4.4)$ & $13(12.4)$ & 0.002 \\
\hline $\begin{array}{l}2 \mathrm{ULN}<\mathrm{ALT}<3 \mathrm{ULN} n \\
(\%)\end{array}$ & $5(1.0)$ & $3(0.7)$ & $2(1.8)$ & 0.292 & $3(0.7)$ & $2(1.9)$ & 0.272 \\
\hline 3ULN<ALT, n (\%) & $2(0.4)$ & $2(0.5)$ & $0(0)$ & 1.000 & $2(0.5)$ & $0(0)$ & 1.000 \\
\hline AST, UI/L & $18(14,22)$ & $18(14,23)$ & $17(14,21)$ & 0.200 & $18(15,23)$ & $17(14,21)$ & 0.080 \\
\hline $\begin{array}{l}\text { ULN < } \text { AST<2ULN, n } \\
(\%)\end{array}$ & $31(6.0)$ & $22(5.5)$ & $9(8.2)$ & 0.288 & $23(5.6)$ & $8(7.6)$ & 0.447 \\
\hline $\begin{array}{l}2 \mathrm{2ULN}<A S T<3 \mathrm{ULN}, \mathrm{n} \\
(\%)\end{array}$ & $2(0.4)$ & $2(0.5)$ & $0(0)$ & 1.000 & $2(0.5)$ & $0(0)$ & 1.000 \\
\hline 3ULN <AST, n (\%) & $2(0.4)$ & $2(0.5)$ & $0(0)$ & 1.000 & $2(0.5)$ & $0(0)$ & 1.000 \\
\hline GGT, UI/L & $22(15,35)$ & $20(14,33)$ & $27(19,43)$ & $<0.001$ & $20(14,33)$ & $27(18,45)$ & $<0.001$ \\
\hline $\begin{array}{l}\text { ULN }<G G T<2 \text { ULN, n } \\
(\%)\end{array}$ & $56(10.9)$ & $40(9.9)$ & $16(14.5)$ & 0.168 & $40(9.8)$ & $16(15.2)$ & 0.111 \\
\hline $\begin{array}{l}2 \mathrm{2ULN}<\mathrm{G} G T<3 \cup L N, n \\
(\%)\end{array}$ & $16(3.1)$ & $12(3.0)$ & $4(3.6)$ & 0.757 & $12(2.9)$ & $4(3.8)$ & 0.752 \\
\hline $3 U L N<G G T, n(\%)$ & $10(1.9)$ & $6(1.5)$ & $4(3.6)$ & 0.233 & $6(1.5)$ & $4(3.8)$ & 0.127 \\
\hline ALP, UI/L & $81(64,99)$ & $81(64,99)$ & $81(64,101)$ & 0.967 & $81(64,99)$ & $81(65,100)$ & 0.949 \\
\hline $\begin{array}{l}\text { ULN }<\text { ALP<2 ULN, n } \\
(\%)\end{array}$ & $25(4.9)$ & $19(4.7)$ & $6(5.5)$ & 0.749 & $19(4.7)$ & $6(5.7)$ & 0.654 \\
\hline $\begin{array}{l}2 \mathrm{ULN}<\mathrm{ALP}<3 \mathrm{ULN}, \mathrm{n} \\
(\%)\end{array}$ & $0(0)$ & $0(0)$ & $0(0)$ & NA & $0(0)$ & $0(0)$ & NA \\
\hline 3ULN<ALP, n (\%) & $0(0)$ & $0(0)$ & $0(0)$ & NA & $0(0)$ & $0(0)$ & NA \\
\hline TBIL, $\mu \mathrm{mol} / \mathrm{L}$ & $\begin{array}{l}8.4 \\
(6.7,10.7)\end{array}$ & $\begin{array}{l}8.6 \\
(6.7,10.9)\end{array}$ & $\begin{array}{l}8.1 \\
(6.7,10.4)\end{array}$ & 0.792 & $\begin{array}{l}8.6 \\
(6.7,10.9)\end{array}$ & $\begin{array}{l}8.1 \\
(6.8,10.4)\end{array}$ & 0.787 \\
\hline $\begin{array}{l}\text { ULN }<\text { TBIL<2 ULN, n } \\
(\%)\end{array}$ & $5(1.0)$ & $4(1.0)$ & $1(0.9)$ & 1.000 & $4(1.0)$ & $1(1.0)$ & 1.000 \\
\hline $\begin{array}{l}2 U L N<T B I L<3 U L N, n \\
(\%)\end{array}$ & $0(0)$ & $0(0)$ & $0(0)$ & NA & $0(0)$ & $0(0)$ & NA \\
\hline 3ULN <TBIL, n (\%) & $0(0)$ & $0(0)$ & $0(0)$ & NA & $0(0)$ & $0(0)$ & NA \\
\hline ALB, g/L & $\begin{array}{l}33.8 \\
(30.7,37.8)\end{array}$ & $\begin{array}{l}33.2 \\
(30.3,37.8)\end{array}$ & $\begin{array}{l}35.0 \\
(31.9,38.3)\end{array}$ & 0.066 & $\begin{array}{l}33.2 \\
(30.3,37.7)\end{array}$ & $\begin{array}{l}35.0 \\
(31.8,38.3)\end{array}$ & 0.084 \\
\hline ALB<LLN, n (\%) & $228(44.4)$ & $181(44.9)$ & 47 (42.7) & 0.683 & $182(44.6)$ & $46(43.8)$ & 0.883 \\
\hline $\begin{array}{l}\text { Abnormal LFTs, } \mathrm{n} \\
(\%)\end{array}$ & $118(23.0)$ & $86(21.3)$ & $32(29.1)$ & 0.087 & $26(6.4)$ & $12(11.4)$ & 0.078 \\
\hline \multicolumn{8}{|l|}{$\begin{array}{l}\text { Hepatic steatosis } \\
\text { scores }\end{array}$} \\
\hline FLI & 11.6 & 8.5 & 27.0 & $<0.001$ & 8.8 & 27.0 & $<0.001$ \\
\hline
\end{tabular}




\begin{tabular}{|c|c|c|c|c|c|c|c|}
\hline & $(4.7,26.1)$ & $(3.9,19.5)$ & $(13.5,53.6)$ & & $(4.0,19.7)$ & $(12.9,54.4)$ & \\
\hline FLI $\geq 60, n(\%)$ & $17(3.3)$ & $0(0)$ & $17(15.5)$ & $<0.001$ & $0(0)$ & $17(16.2)$ & $<0.001$ \\
\hline \multicolumn{8}{|l|}{ Liver fibrosis score } \\
\hline FIB-4 & $\begin{array}{l}0.80 \\
(0.54,1.15)\end{array}$ & $\begin{array}{l}0.80 \\
(0.53,1.15)\end{array}$ & $\begin{array}{l}0.82 \\
(0.59,1.14)\end{array}$ & 0.504 & $\begin{array}{l}0.80 \\
(0.53,1.17)\end{array}$ & $\begin{array}{l}0.80 \\
(0.57,1.05)\end{array}$ & 0.857 \\
\hline $\begin{array}{l}\text { Advanced fibrosis, } n \\
(\%)\end{array}$ & $70(13.6)$ & $54(13.4)$ & $16(14.5)$ & 0.756 & $58(14.2)$ & $12(11.4)$ & 0.458 \\
\hline NFS & $\begin{array}{l}-2.09 \\
(-3.37,-1.03)\end{array}$ & $\begin{array}{l}-2.19 \\
(-3.50,-1.23)\end{array}$ & $\begin{array}{l}-1.56 \\
(-2.66,-0.38)\end{array}$ & $<0.001$ & $\begin{array}{l}-2.17 \\
(-3.49,-1.20)\end{array}$ & $\begin{array}{l}-1.62 \\
(-2.74,-0.46)\end{array}$ & 0.001 \\
\hline $\begin{array}{l}\text { Advanced fibrosis, } n \\
(\%)\end{array}$ & $104(20.3)$ & $64(15.9)$ & $40(36.4)$ & $<0.001$ & $66(16.2)$ & $38(36.2)$ & $<0.001$ \\
\hline Forns index & $\begin{array}{l}4.89 \\
(3.83,5.82)\end{array}$ & $\begin{array}{l}4.85 \\
(3.73,5.76)\end{array}$ & $\begin{array}{l}5.19 \\
(4.24,6.18)\end{array}$ & 0.008 & $\begin{array}{l}4.86 \\
(3.74,5.78)\end{array}$ & $\begin{array}{l}5.02 \\
(4.16,6.15)\end{array}$ & 0.040 \\
\hline $\begin{array}{l}\text { Advanced fibrosis, } n \\
(\%)\end{array}$ & $128(25.0)$ & $91(22.6)$ & $37(33.6)$ & 0.018 & $95(23.3)$ & 33 (31.4) & 0.085 \\
\hline
\end{tabular}

ALT, alanine aminotransferase; AST, aspartate aminotransferase; GGT, y-glutamyl transferase; ALP, alkaline phosphatase; TBIL, total bilirubin; ALB, albumin; ULN, upper limit of normal; LLN, lower limit of normal; LFTs, liver function tests; FLI, fatty liver index; FIB-4, fibrosis-4 index; NFS, NAFLD fibrosis score

* Compared between RA patients with or without MAFLD

\# Compared between RA patients with or without NAFLD

Table 3

Comparisons of CVD events and 10-year CVD risk between RA patients with and without MAFLD or NAFLD 


\begin{tabular}{|c|c|c|c|c|c|c|c|}
\hline Characteristics & $\begin{array}{l}\text { RA } \\
\text { patients } \\
(n=513)\end{array}$ & $\begin{array}{l}\text { No- } \\
\text { MAFLD } \\
(n=403)\end{array}$ & $\begin{array}{l}\text { MAFLD } \\
(n=110)\end{array}$ & $P^{*}$ & $\begin{array}{l}\text { No- } \\
\text { NAFLD } \\
(n=408)\end{array}$ & $\begin{array}{l}\text { NAFLD } \\
(n=105)\end{array}$ & $P^{\#}$ \\
\hline CVD events, n (\%) & $56(10.9)$ & $37(9.2)$ & 19 (17.3) & 0.016 & $37(9.1)$ & $19(18.1)$ & 0.008 \\
\hline Myocardial infarction, n (\%) & $12(2.3)$ & $6(1.5)$ & $6(5.5)$ & 0.025 & $6(1.5)$ & $6(5.7)$ & 0.020 \\
\hline Angina pectoris, n (\%) & $7(1.4)$ & $3(0.7)$ & $4(3.6)$ & 0.041 & $3(0.7)$ & $4(3.8)$ & 0.035 \\
\hline Heart failure, n (\%) & $19(3.7)$ & $14(3.5)$ & $5(4.5)$ & 0.574 & $14(3.4)$ & $5(4.8)$ & 0.561 \\
\hline Ischemic stroke, n (\%) & $23(4.5)$ & $18(4.5)$ & $5(4.5)$ & 1.000 & $18(4.4)$ & $5(4.8)$ & 0.796 \\
\hline Hemorrhagic stroke, n (\%) & $0(0)$ & $0(0)$ & $0(0)$ & NA & $0(0)$ & $0(0)$ & NA \\
\hline $\begin{array}{l}\text { Peripheral arterial disease, } \\
\mathrm{n}(\%)\end{array}$ & $3(0.6)$ & $1(0.2)$ & $2(1.8)$ & 0.118 & $1(0.2)$ & $2(1.9)$ & 0.108 \\
\hline \multicolumn{8}{|l|}{ 10-year CVD risk } \\
\hline SCORE for low-risk region & $\begin{array}{l}0.0 \\
(0.0,3.0)\end{array}$ & $\begin{array}{l}0.0 \\
(0.0,1.5)\end{array}$ & $\begin{array}{l}1.5 \\
(0.0,3.0)\end{array}$ & 0.023 & $\begin{array}{l}0.0 \\
(0.0,1.5)\end{array}$ & $\begin{array}{l}1.5 \\
(0.0,3.0)\end{array}$ & 0.039 \\
\hline SCORE for high-risk region & $\begin{array}{l}1.5 \\
(0.0,4.5)\end{array}$ & $\begin{array}{l}1.5 \\
(0.0,4.5)\end{array}$ & $\begin{array}{l}1.5 \\
(1.5,4.5)\end{array}$ & 0.003 & $\begin{array}{l}1.5 \\
(0.0,4.5)\end{array}$ & $\begin{array}{l}1.5 \\
(1.5,4.5)\end{array}$ & 0.005 \\
\hline FRS & $\begin{array}{l}1.5 \\
(0.0,7.5)\end{array}$ & $\begin{array}{l}1.5 \\
(0.0,7.5)\end{array}$ & $\begin{array}{l}3.0 \\
(1.5,6.0)\end{array}$ & 0.002 & $\begin{array}{l}1.5 \\
(0.0,7.5)\end{array}$ & $\begin{array}{l}3.0 \\
(1.5,6.0)\end{array}$ & 0.002 \\
\hline China-PAR Equation & $\begin{array}{l}2.5 \\
(0.9,6.0)\end{array}$ & $\begin{array}{l}2.1 \\
(0.8,5.8)\end{array}$ & $\begin{array}{l}3.7 \\
(2 \cdot 0,7.7)\end{array}$ & $<0.001$ & $\begin{array}{l}2.1 \\
(0.8,5.9)\end{array}$ & $\begin{array}{l}3.7 \\
(2 \cdot 0,7.7)\end{array}$ & $<0.001$ \\
\hline$\underset{(\%)}{\text { High 10-year CVD risk, n }}$ & $166(32.4)$ & $105(26.1)$ & $61(55.5)$ & $<0.001$ & $\begin{array}{l}108 \\
(26.5)\end{array}$ & $58(55.2)$ & $<0.001$ \\
\hline
\end{tabular}

CVD, Cardiovascular disease; SCORE, the Systemic Coronary Risk Evaluation; FRS, Framingham risk score

China-PAR, the Prediction for ASCVD Risk in China

* Compared between RA patients with or without MAFLD

\# Compared between RA patients with or without NAFLD

Table 4

Logistic regression analysis for potential associated factors of CVD events and high 10-year CVD risk in RA patients 


\begin{tabular}{|c|c|c|c|c|c|}
\hline \multirow[t]{2}{*}{ Characteristics } & & \multicolumn{2}{|l|}{ Univariate } & \multicolumn{2}{|l|}{ Multivariate } \\
\hline & & OR $(95 \% C l)$ & $P$ & $\operatorname{AOR}(95 \% C l)$ & $P$ \\
\hline CVD events & $\mathrm{n}(\%)$ & & & & \\
\hline \multicolumn{6}{|l|}{ NAFLD } \\
\hline No-NAFLD & $37(9.1)$ & Ref & Ref & Ref & Ref \\
\hline NAFLD & $19(18.1)$ & $2.215(1.215,4.040)$ & 0.009 & $2.478(1.185,4.779)$ & 0.007 \\
\hline \multicolumn{6}{|l|}{ MAFLD } \\
\hline No-MAFLD & $37(9.2)$ & Ref & Ref & Ref & Ref \\
\hline MAFLD & 19 (17.3) & $2.065(1.135,3.759)$ & 0.018 & $2.303(1.197,4.429)$ & 0.012 \\
\hline \multicolumn{6}{|l|}{ Three subtypes of MAFLD } \\
\hline No-MAFLD & $37(9.2)$ & Ref & Ref & NA & NA \\
\hline MAFLD (overweight/obesity) & $7(14.6)$ & $1.689(0.708,4.031)$ & 0.238 & NA & NA \\
\hline MAFLD (lean/normal weight) & $4(14.3)$ & $1.649(0.543,5.009)$ & 0.378 & NA & NA \\
\hline MAFLD (T2DM) & $8(23.5)$ & $3.044(1.286,7.204)$ & 0.011 & NA & NA \\
\hline High 10-year CVD risk & $\mathrm{n}(\%)$ & & & & \\
\hline \multicolumn{6}{|l|}{ NAFLD } \\
\hline No-NAFLD & $108(26.5)$ & Ref & Ref & Ref & Ref \\
\hline NAFLD & $58(55.2)$ & $3.428(2.201,5.339)$ & $<0.001$ & $2.870(1.597,5.156)$ & $<0.001$ \\
\hline \multicolumn{6}{|l|}{ MAFLD } \\
\hline No-MAFLD & $105(26.1)$ & Ref & Ref & Ref & Ref \\
\hline MAFLD & $61(55.5)$ & $3.533(2.283,5.469)$ & $<0.001$ & $3.184(1.777,5.705)$ & $<0.001$ \\
\hline
\end{tabular}

AOR adjusted by age, sex, smoking habits, RA disease duration, positive RF, positive ACPA, CDAI, HAQ-DI, mTSS, previous treatment, BMI, BP, FPG, TC, TG, HDL-C and LDL-C

NA: Unsuitable due to the small sample

\section{Figures}

\section{Figure 1}

The prevalence of MAFLD and NAFLD in RA patients with different stratification.

The prevalence and standardized prevalence in different genders (A, C) and ages groups (B, D); The prevalence in different disease activity groups $(E)$ and disease duration $(F)$; The standardized prevalence of MAFLD and NAFLD was adjusted the age or sex composition ratio according to the data in the 2020 China Statistical Yearbook; remission (CDAl $\leq 2.8)$; active $(\mathrm{CDAl}>2.8)$ 


\section{Supplementary Files}

This is a list of supplementary files associated with this preprint. Click to download.

- Supplementarymaterials.docx 